\title{
Predicting Knee Pain and Knee Osteoarthritis Among Overweight Women
}

\author{
Marieke L. A. Landsmeer, MD, Jos Runhaar, PhD, Marienke van Middelkoop, PhD, \\ Edwin H. G. Oei, MD, PhD, Dieuwke Schiphof, PhD, PatrickJ. E. Bindels, MD, PhD, \\ and Sita M. A. Bierma-Zeinstra, PhD
}

Background: There is a need for prediction of knee osteoarthritis (KOA) in general practice to motivate subjects for preventive therapies and optimize preventive trials.

Aim: To develop a prediction model, with questionnaire and physical examination variables, for incident frequent knee pain (FKP) and symptomatic KOA after 2.5 and/or 6.5 years among overweight and obese middle-aged women.

Design and Setting: Models were developed in the Prevention of Knee Osteoarthritis in Overweight Females study (age 50 to 60 years, body mass index $[\mathrm{BMI}] \geq 27 \mathrm{~kg} / \mathrm{m}^{2}$ ) (ISRCTN 42823086 ). FKP was defined as knee pain during most days in the past month. Symptomatic KOA was defined according to the combined (clinical and radiographic) American College of Rheumatology criteria.

Method: Multivariable analysis by backward stepwise deletion was performed for questionnaire and physical examination variables. The prediction model was externally validated in Rotterdam Study (RS)III. Area under the curves (AUCs) of receiver operating characteristic were calculated.

Results: $32 \%$ of 237 women (mean age $55.7 \pm 3.2$ years; mean BMI, $31.9 \pm 3.8 \mathrm{~kg} / \mathrm{m}^{2}$ ) developed FKP and $30 \%$ developed symptomatic KOA. AUC of age and BMI was $0.63(0.55$ to 0.71$)$ for incident FKP. The final model included age, BMI, mild knee symptoms, knee problems climbing stairs, morning stiffness, postmenopausal status, and heavy work. AUC was $0.71(0.63$ to 0.78$)$. Results were similar for incident KOA. Applying external validation, similar results were observed in the RS-III.

Conclusion: In this study, easy-obtainable variables modestly improved the prediction of FKP and symptomatic KOA above age and BMI. To improve the identification of high-risk individuals, development of valid tests for other known risk factors, like meniscal damage, that are applicable in primary care, are urgently needed. (J Am Board Fam Med 2019;32:575-584.)

Keywords: Body Mass Index, Chronic Disease, Netherlands, Obesity, Osteoarthritis of Knee, Pain, Primary Health Care, Rheumatology

Frequent knee pain (FKP) and knee osteoarthritis $(\mathrm{KOA})$ are common complaints in general practice and constitute a substantial workload. ${ }^{1,2}$ Knee pain affects $\pm 25 \%$ in those over 55 years of age; more

This article was externally peer reviewed.

Submitted 11 October 2018; revised 29 January 2019; accepted 5 February 2019.

From the Department of General Practice, Erasmus MC, University Medical Center Rotterdam, the Netherlands (MLAL, JR, MvM, DS, PJEB, SMABZ); Department of Radiology \& Nuclear Medicine, Erasmus MC, University Medical Center Rotterdam, the Netherlands (EHGO); Department of Orthopaedics, Erasmus MC, University Medical Center Rotterdam, the Netherlands (SMABZ).

Funding: The PROOF study has been funded by ZonMW, the Netherlands Organisation for Health Research and Development (Grant 120520001). It has received partial funding women than men. ${ }^{1} \mathrm{KOA}$ is the most frequent diagnosis associated with FKP in older people. ${ }^{3}$

In primary care, attention for prevention of diseases is common. Due to the large individual and

from a program grant of the Dutch Arthritis foundation for their centre of excellence "Osteoarthritis in primary care" (Grant LLP19). The D-Board project has received funding from the European Union's Seventh Framework Programme for research, technological development and demonstration under Grant Agreement 305815. None of the funding sources had a role in the study design, collection, analysis or interpretation of data, in the writing of the manuscript or in the decision to submit the manuscript for publication.

Conflict of interest: none declared.

Corresponding author: Marieke L. A. Landsmeer, MD, Erasmus MC, Department of General Practice, Office Na 1818, P.O. Box 2040, 3000 CA Rotterdam, the Netherlands (E-mail: m.landsmeer@erasmusmc.nl). 
socioeconomic burden of KOA and FKP, there is growing interest in early detection and prevention. ${ }^{1,4}$ Although overweight is one of the most important risk factors, other factors might also predict FKP or symptomatic KOA. ${ }^{5}$ Identification of high-risk subjects is necessary for 2 reasons. First, to offer and motivate them for preventive strategies such as changes in lifestyle and in occupational habits. Heightening risk appraisals might change people's intentions and behavior, as shown in literature, ${ }^{6}$ without leading to unintended adverse effects. ${ }^{7,8}$ Second, identification is necessary to optimize preventive trials in osteoarthritis (OA) research in a most cost-effective manner by including subjects in a study who are at highest risk of developing FKP/KOA on a short term.

Several studies have investigated prediction of KOA. ${ }^{9-11}$ Zhang et $\mathrm{al}^{10}$ developed a model with conventional and modifiable risk factors for the prediction of symptomatic radiographic KOA in a general population aged 40 to 70 years. This model, incorporating age, gender, body mass index (BMI), occupational kneeling/lifting, family history, and knee injury, resulted in an area under the curve (AUC) of $0.70 .{ }^{10}$ Kerkhof et $\mathrm{al}^{9}$ developed a model for the prediction of radiographic KOA in a general population aged 55 years and over, incorporating age, gender, BMI, questionnaire variables, genetic score, a urinary biomarker, and radiographic signs of possible osteophytes (Kellgren and Lawrence $[\mathrm{KL}]$ ) grade $1^{12}$ ). Questionnaire variables, genetic score or a urinary biomarker did not improve prediction versus age, gender, and $\mathrm{BMI}$ (AUC, 0.66). The AUC increased to 0.79 by adding baseline KL 1. ${ }^{9}$ Sharma et al ${ }^{11}$ developed a model for prediction of radiographic KOA among persons at higher risk for KOA but with KL 0 in both knees (no radiographic KOA). They found that Magnetic Resonance Imaging (MRI) lesions in tissues known to be involved in KOA improved prediction when added to models including age, gender, BMI, hand $\mathrm{OA}$, injury, surgery, occupational activity, and knee symptoms or function (AUC, 0.84). ${ }^{11}$

For a general practitioner (GP) however, the use of easily obtainable variables without the need for additional laboratory or radiologic assessments is highly preferable. It is known that the most important risk factors for incident knee pain/symptomatic KOA are older age, female gender, and overweight/obesity. However, even in such a high-risk population, not all women develop KOA, as shown by the preventive trial of Runhaar et al. ${ }^{13}$ Therefore, it would be interesting to investigate predictors within such a high-risk group. ${ }^{14}$ In addition, up to now, no studies have evaluated prediction of FKP, while this symptom is more important for patients than the underlying pathology (structural $\mathrm{KOA}){ }^{15,16}$

Therefore, the objective of this study was to develop a risk prediction model for the development of FKP and symptomatic KOA in general practice among overweight middle-aged women incorporating questionnaire and physical examination variables.

\section{Method}

\section{Study Design and Population}

We used data from the Prevention of Knee Osteoarthritis in Overweight Females (PROOF) study, ${ }^{13}$ a randomized controlled trial in general practices in the Rotterdam area, the Netherlands. PROOF evaluated the preventive effects of a tailor-made diet and exercise program and of oral glucosamine sulfate versus placebo on the development of KOA over 2.5 years in 407 overweight and obese women of 50 to 60 years (ISRCTN 42823086). ${ }^{13}$ Post-hoc long-term outcome evaluation was performed over 6.5 years' followup (mean, $6.7 \pm 0.7$ years). The Institutional Review Board of Erasmus University Medical Center approved the study and participants gave written informed consent. Participants were recruited by their GP by sending study information and a reply card to all registered women between 50 and 60 years. They had to be free of KOA according to the clinical American College of Rheumatology (ACR) criteria. ${ }^{17}$ Further inclusion criteria were $B M I \geq 27 \mathrm{~kg} / \mathrm{m}^{2}$, no inflammatory rheumatic diseases, no severely disabling comorbidities, not under treatment of a physical therapist or GP for knee complaints, not using walking aids, not using oral glucosamine for the past 6 months, and mastering of the Dutch language. At baseline, participants filled in a questionnaire and underwent standardized physical examination at the research institute. Baseline posterior-anterior radiographs of both knees were taken using the semiflexed metatarsophalangeal protocol. ${ }^{18}$ Measurements were repeated after 2.5 and 6.5 years. Only women participating at 2.5 years were asked to participate at 6.5 years. 


\section{Risk Factor Assessment}

Candidate predictors were selected on litera-

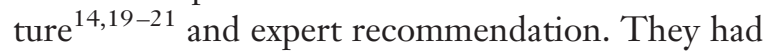
to be easily obtainable through history taking or physical examination. We identified 16 relevant variables for the prediction of FKP (pain in or around 1 or both knees during most days in the past month $^{22}$ ) and symptomatic KOA.

\section{Questionnaire Variables}

Age, assessed by questionnaire. Postmenopausal status was defined after 12 consecutive months of amenorrhoea. Depression was defined when diagnosed with depression or having depressive complaints during the previous 3 months. Family history of OA (self reported) was present when at least 1 first-degree relative had OA. Injury was defined when the women had ever visited a doctor for knee injury (no/yes). Physically demanding work was defined as doing heavy physical work "quite often" or "(almost) always." Mild knee symptoms were assessed using the question, "Did you experience any pain in or around your knee within the past 12 months?" (no/yes). Instability of the knee was assessed with the question, "Did you experience a sensation of the knee giving way within the past 12 months?"(no/yes). "Knee problems while climbing stairs" and "knee problems while standing up from a chair," defined with the Knee Injury and Osteoarthritis Outcome Score (KOOS) subscale on "Function, daily living," ${ }^{23}$ were present when the women had any (seldom/sometimes/often/always) physical limitation due to her knee while climbing stairs or standing up from a chair, respectively. Morning stiffness, evaluated with KOOS subscale on "Stiffness," ${ }^{23}$ was present when the women had moderate/much/very much knee joint stiffness after sleeping (vs no/little). Swelling of the knee, assessed with KOOS subscale on "Symptoms," was present when the women had any swelling of the knee during the last week. The cutoff points for the KOOS variables were based on their distribution, with $10 \%$ as lower limit.

\section{Physical Examination Variables}

Body height in standing position without shoes and weight were measured at baseline examination; BMI was calculated $\left(\mathrm{kg} / \mathrm{m}^{2}\right)$. Both hands were examined for Heberden's nodes (no/yes). Both knees were examined for pain at palpation of the medial and lateral joint line (no/yes) and tested for crepi- tus $^{21}$ during active flexion and extension of the knee (no/yes).

\section{Outcome Measure Assessment}

Outcome measures were incident FKP and incident symptomatic KOA after 2.5 and/or 6.5 years. FKP was assessed by questionnaire ("Did you experience pain in or around 1 or both knees during most days in the past month?" [no/yes]). Incident FKP after 2.5 and/or 6.5 years was defined when present at 2.5 and/or 6.5 years and not present at baseline. Symptomatic KOA was defined according to the combined (clinical and radiographic) ACR criteria ${ }^{17}$ : FKP and a definite tibiofemoral osteophyte in the same knee and one of the following: age $>50$ years, morning stiffness $<30$ minutes, crepitus on active knee motion. Incident symptomatic KOA after 2.5 and/or 6.5 years was defined when present at 2.5 and/or 6.5 years and not present at baseline.

\section{Statistical Analysis}

Analyses were done using the subject as unit of analysis. Descriptive data were presented as mean \pm standard deviation (SD) or as counts (percentages). Age and BMI were kept continuous, other predictors were dichotomous. Preliminary analyses were conducted to ensure no violation of the multicollinearity assumption. Tolerance values were $>0.6$ for all predictors. For each outcome a binary logistic regression model was created with age and BMI (basic model). Next, all potential predictors were analyzed in a multivariable logistic regression model for each outcome. Backward stepwise deletion based on the Wald test was applied using a $P$ value of .20 , to reduce the number of predictors in the final model. Since study participants were part of a randomized trial, analyses were additionally run with adjustments for the original randomization groups and their interaction. ${ }^{13}$ The HosmerLemeshow $\chi^{2}$ statistics for goodness-of-fit were used to compare observed and predicted risks. The explained variance was assessed by the Nagelkerke $\mathrm{R}^{2}$. Discriminative ability was assessed with the AUC of the receiver operating characteristic. Analyses were performed with SPSS 21.0 (Chicago, IL). $P<.05$ were considered statistically significant.

\section{External Validation}

For external validation of the final model, data from the Rotterdam Study (RS) were used. The RS 
is a population-based cohort study in the Netherlands that investigates determinants, incidence, and progression of chronic disabling diseases in the elderly. ${ }^{24}$ We used data of the RS-III-1 subcohort. Apart from "physically demanding work," risk factor assessment was identical in the RS and in PROOF. In the RS, "physically demanding work" was defined as doing intense work (regularly lifting heavy objects at work) (no/yes) obtained with the Short Questionnaire to Assess Health-Enhancing Physical Activity $\left(\mathrm{SQUASH}^{25}\right)$. Women aged 50 to 60 years with $B M I \geq 27 \mathrm{~kg} / \mathrm{m}^{2}$ were included for validation. Mean follow-up of RS-III-1 was $4.62 \pm$ 0.56 years. $^{21}$ The medical ethics committee of Erasmus University Medical Center approved the study and participants provided written consent.

\section{Results}

\section{Study Population}

Thirty-six of 407 women (9\%) with baseline FKP were excluded for analyses, and 134 of 371 women (36\%) had missing data for baseline, 2.5 , or 6.5 years FKP questions. Reasons were not completing the FKP questions $(\mathrm{n}=7)$, unattainability $(\mathrm{n}=8)$, no further time available or interest in the study $(n=114)$. Five women died (not related to the study outcomes). For the prediction of symptomatic KOA, 24 women (6\%) were excluded due to baseline symptomatic KOA, and 148 of 383 women (39\%) had missing data for FKP ( $\mathrm{n}=134)$ or for radiography data $(n=14)$.

Table 1 describes the baseline characteristics of both study populations. There were only $0.8 \%$ missing values of predictor variables. Dropouts based on missing outcome had a lower presence of "family history of OA" compared with those with complete outcome (35\% vs $48 \%$ for FKP, $38 \%$ vs $49 \%$ for symptomatic KOA).

Seventy-five of 237 women (32\%) developed FKP and 70 of 235 (30\%) symptomatic KOA. Within those with incident FKP $(\mathrm{n}=75)$, 93\% $(\mathrm{n}=70)$ had a tibiofemoral osteophyte ipsilateral, hence also fulfilled the combined ACR criteria. Given the large overlap between the 2 cohorts, the results of the incident symptomatic KOA cohort are presented in Appendix Table 1.

\section{Risk Prediction Models}

In the final model for incident FKP, the following predictors were selected based on our selection criteria: age, BMI, mild knee symptoms, knee problems while climbing stairs, morning stiffness, postmenopausal status, and physically demanding work (Table 2). When the analysis was additionally corrected for the original trial interventions and their interaction, minor nonrelevant changes were found (data not shown).

\section{Internal Validation}

Internal validation showed good calibration for the basic and final prediction model for incident FKP (Table 2). The basic model showed an AUC of 0.63 (0.55 to 0.71$)$. Prediction improved in the final model to an AUC of 0.71 (0.63 to 0.78).

\section{External Validation}

In RS-III-1, 346 women were 50 to 60 years with $\mathrm{BMI} \geq 27 \mathrm{~kg} / \mathrm{m}^{2}$. Two hundred thirty-six of 346 women $(68 \%)$ had data available for the prediction of FKP and 264 (76\%) for the prediction of symptomatic KOA. In the FKP cohort (mean age, $55.4 \pm 3.2$ years; mean BMI, $\left.30.8 \pm 3.5 \mathrm{~kg} / \mathrm{m}^{2}\right), 41$ (17\%) developed FKP. In the symptomatic KOA cohort (mean age, $55.5 \pm 3.3$ years; mean BMI, $\left.30.9 \pm 3.6 \mathrm{~kg} / \mathrm{m}^{2}\right), 19(7 \%)$ developed symptomatic KOA. The AUC for the prediction of FKP was 0.71 (0.62 to 0.79 ) (Table 3). For the prediction of symptomatic KOA, the AUC was 0.81 (0.72 to 0.90) (Appendix Table 2).

\section{Discussion \\ Summary}

We aimed to develop a risk prediction model for GPs with easily obtainable predictors for incident FKP and incident symptomatic KOA among overweight and obese middle-aged women. A basic model, with only age and BMI had little discriminative power. With the variables age, BMI, mild knee symptoms, knee problems while climbing stairs, morning stiffness, postmenopausal status, and physically demanding work, the AUC of the prediction model for incident FKP increased to 0.71 ( 0.63 to 0.78$)$. Similar results were found for the prediction model of incident symptomatic KOA.

\section{Comparison with Existing Literature}

Besides age and BMI, several variables were selected in the final prediction model for FKP, among which mild knee symptoms, problems while 


\begin{tabular}{|c|c|c|}
\hline Baseline Characteristics & $\begin{array}{l}\text { Study Population for } \\
\text { Incident Frequent } \\
\text { Knee Pain } \$ \$ \\
(\mathrm{n}=237)\end{array}$ & $\begin{array}{l}\text { Study Population for Incident } \\
\text { Symptomatic Knee OA } \\
\qquad(\mathrm{n}=235)\end{array}$ \\
\hline \multicolumn{3}{|l|}{ Questionnaire variables } \\
\hline Age (years), mean (SD) & $55.7 \pm 3.2$ & $55.8 \pm 3.2$ \\
\hline BMI $\left(\mathrm{kg} / \mathrm{m}^{2}\right)$, mean $(\mathrm{SD})$ & $31.9 \pm 3.8$ & $31.9 \pm 3.8$ \\
\hline Postmenopausal status, n (\%) & $159(67)$ & $159(68)$ \\
\hline Comorbidity of depression*, n (\%) & $16(7)$ & $16(7)$ \\
\hline Family history of $\mathrm{OA}^{\dagger}, \mathrm{n}(\%)$ & $113(48)$ & $112(48)$ \\
\hline History of knee injury ${ }^{\ddagger}, \mathrm{n}(\%)$ & $46(19)$ & $47(20)$ \\
\hline Physically demanding work ${ }^{\S}, \mathrm{n}(\%)$ & $24(10)$ & $24(10)$ \\
\hline Mild knee symptoms" ${ }^{\mathbb{I}}$, (\%) & $98(41)$ & $100(43)$ \\
\hline Feeling of giving way", $\mathrm{n}(\%)$ & $35(15)$ & $37(16)$ \\
\hline Knee problems while climbing stairs ${ }^{* *}$, n (\%) & $20(8)$ & $20(9)$ \\
\hline Knee problems standing up from chair**, n (\%) & $56(24)$ & $58(25)$ \\
\hline Morning stiffness ${ }^{\dagger \dagger}, \mathrm{n}(\%)$ & $29(12)$ & $29(12)$ \\
\hline Swollen knee & $27(11)$ & $27(11)$ \\
\hline \multicolumn{3}{|l|}{ Physical examination variables } \\
\hline Heberden's nodes (in $\geq 1$ finger), $\mathrm{n}(\%)$ & $65(27)$ & $64(27)$ \\
\hline Joint line tenderness (medial and/or lateral), n (\%) & $26(11)$ & $25(11)$ \\
\hline Crepitus during active motion, $\mathrm{n}(\%)$ & $134(57)$ & $135(57)$ \\
\hline
\end{tabular}

BMI, body mass index; N, number of women; OA, Osteoarthritis; SD, standard deviation.

${ }^{*}$ Comorbidity of depression was defined as being diagnosed with depression and/or currently under treatment.

${ }^{\dagger}$ Present when at least one first-degree relative had OA.

${ }^{\ddagger}$ Present when visited a doctor for a knee injury.

${ }^{\S}$ Doing heavy physical work "quite often" or "(almost) always".

"Pain in or around the knee within the last 12 months.

"The sensation of the knee giving way within the last 12 months.

${ }^{* *}$ Defined with the Knee Injury and Osteoarthritis Outcome Score (KOOS) on physical functioning.

${ }^{+\dagger}$ Defined with the KOOS on stiffness.

${ }^{\ddagger \neq}$ Defined with the KOOS on symptoms.

${ }^{\$}$ Incident frequent knee pain after 2.5 and/or 6.5 years was defined when frequent knee pain was present at 2.5 and/or 6.5 years and when no knee pain was reported at baseline. Frequent knee pain was defined as self-reported pain in or around one or both knees during most days in the past month.

"IIIncident symptomatic knee OA after 2.5 and/or 6.5 years was defined when incident symptomatic knee OA was present at 2.5 and/or 6.5 years and not present at baseline. Symptomatic knee OA was defined according to the clinical and radiographic ACR criteria: self-reported frequent knee pain and a definite osteophyte in the tibiofemoral joint of the same knee and one of the following: age $>50$ years, morning stiffness $<30$ minutes, crepitus on active motion of the knee.

climbing stairs, morning stiffness, and physically demanding work. These factors are also found in other studies: the Osteoarthritis Initiative showed that pain during weight-bearing, knee-bending activities like climbing stairs, could be used to identify early OA. ${ }^{26}$ In addition, studies showed that increased risk of chronic knee pain was found among occupations that involve knee bending and heavy lifting. ${ }^{27,28}$ Recently, a proposal for classification criteria for early knee OA has been published, including stiffness as a symptom of early knee OA. ${ }^{29}$ The incidence of developing FKP in the present study was $32 \%$. This increases to $47 \%$ ("post-test") when knee problems while climbing stairs are pres- ent and to $52 \%$ when morning stiffness is present. None of the physical examination variables, except BMI, were selected in the final model. The only one study that evaluated physical examination variables for the prediction of KOA was the study by Sharma et al. ${ }^{11}$ In their study, "Heberden's nodes" was selected in their final prediction model by selection of univariable significance $(P<.1)$. Joint line tenderness and crepitus were not examined in their study. ${ }^{11}$ Although our final model improved the basic model, the overall explained variance is still low and suggests that prediction of FKP (and symptomatic KOA) with easily obtainable risk factors seems not yet clinically applicable. 
Table 2. Multivariable Models in Prediction of Incident Frequent Knee Pain and Internal Validation (Calibration and Discrimination) of the Risk Prediction Models

\begin{tabular}{|c|c|c|c|c|}
\hline \multirow[b]{2}{*}{ Selected Predictors } & \multicolumn{2}{|c|}{ Basic Model } & \multicolumn{2}{|c|}{ Backward Model } \\
\hline & OR $(95 \% \mathrm{CI})$ & $P$ Value & OR $(95 \% \mathrm{CI})$ & $P$ Value \\
\hline Age (years) & $1.07(0.98-1.16)$ & .16 & $1.15(1.03-1.28)$ & .02 \\
\hline $\mathrm{BMI}\left(\mathrm{kg} / \mathrm{m}^{2}\right)$ & $1.09(1.01-1.17)$ & .02 & $1.13(1.04-1.23)$ & .004 \\
\hline Mild knee symptoms* & & & $1.74(0.88-3.44)$ & .12 \\
\hline Knee problems while climbing stairs ${ }^{\dagger}$ & & & $2.06(1.03-4.12)$ & .04 \\
\hline Morning stiffness ${ }^{\ddagger}$ & & & $3.03(1.17-7.81)$ & .02 \\
\hline Postmenopausal status & & & $0.57(0.28-1.18)$ & .13 \\
\hline Physically demanding work ${ }^{\S}$ & & & $2.05(0.72-5.83)$ & .18 \\
\hline AUC of the model & $0.63(0.55-0.71)$ & & $0.71(0.63-0.78)$ & \\
\hline Calibration: Hosmer-Lemeshow $P$ value & .72 & & .93 & \\
\hline Variance explained (Nagelkerke), \% & 4.6 & & 21.0 & \\
\hline
\end{tabular}

AUC, area under the curve of the receiver operating characteristic; BMI, body mass index; CI, confidential interval; OR, odds ratio. Bold indicates $P<.05$.

*Pain in or around the knee within the last 12 months.

${ }^{\dagger}$ Defined with the Knee Injury and Osteoarthritis Outcome Score (KOOS) on physical functioning.

${ }^{\ddagger}$ Defined with the KOOS on stiffness.

${ }^{\S}$ Doing heavy physical work "quite often" or "(almost) always".

Incidence rates in the present study are higher than in population-based cohorts, ${ }^{9,30}$ but comparable to rates found among overweight subjects. ${ }^{31,32}$ In RSIII-1, incidence of FKP (17\%) and symptomatic $\mathrm{KOA}(7 \%)$ was lower than in PROOF. This might be explained by lower baseline BMI and prevalence of mild knee symptoms in RS-III-1. In addition, no $\mathrm{X}$-rays were performed during an intermediate assessment, as done in PROOF after 2.5 years. Hence, incidence was based on the outcome after \pm 5 years.
As seen in Appendix Table 2, we found a higher AUC in RS-III-1 (0.81 [0.72 to 0.90]) than in the PROOF study (0.72 [0.64 to 0.80]) for prediction of symptomatic KOA, due to the strong association between "knee problems while climbing stairs" and incident symptomatic KOA (OR, 4.47 [1.31 to 15.23]) in RSIII-1. In addition, the baseline prevalence of "knee problems while climbing stairs" was higher in RSIII-1 (30\%) than in PROOF (9\%). This both resulted in better prediction within RS-III-1.

Table 3. External Validation for the Prediction of Incident Frequent Knee Pain in Rotterdam Study-III-1

\begin{tabular}{lcc}
\hline & \multicolumn{1}{c}{ Study Population $(\mathrm{n}=236)$} \\
\cline { 2 - 3 } Candidate Predictor & OR (95\% CI) & .88 \\
\hline Age (years) & $1.01(0.89-1.15)$ & .13 \\
BMI $\left(\mathrm{kg} / \mathrm{m}^{2}\right)$ & $1.08(0.98-1.18)$ & .13 \\
Mild knee symptoms* & $1.80(0.84-3.87)$ & .07 \\
Knee problems while climbing stairs $^{\dagger}$ & $2.14(0.95-4.82)$ & .29 \\
Morning stiffness $^{\ddagger}$ & $1.65(0.66-4.16)$ & .96 \\
Postmenopausal status $_{\text {Physically demanding work }}^{\S}$ & $0.98(0.36-2.69)$ & .76 \\
AUC of the model & $1.14(0.49-2.66)$ & .57 \\
Calibration: Hosmer-Lemeshow $P$ value & $0.71(0.62-0.79)$ & 12.2 \\
Variance explained (Nagelkerke), \% & & \\
\hline
\end{tabular}

AUC, area under the curve of the receiver operating characteristic; BMI, body mass index; CI, confidential interval; OR, odds ratio. *Pain in or around the knee within the last 12 months.

${ }^{\dagger}$ Defined with the Knee Injury and Osteoarthritis Outcome Score (KOOS) on physical functioning.

${ }^{\ddagger}$ Defined with the KOOS on stiffness.

${ }^{\S}$ Defined as doing intense work (regularly lifting heavy objects at work). 


\section{Strengths and Limitations}

With the PROOF study, the first preventive randomized controlled trial in KOA, the first steps in preventive research within a high-risk population for KOA, have been made. ${ }^{13}$ The present study is directly applicable to GPs, since only easily obtainable variables were used for the prediction models and also a symptom-only definition was used. Moreover, models were externally validated, which confirmed our results.

There are also some limitations. High numbers lost to followup might have introduced selection bias. There were no significant differences in baseline variables compared with complete cases, except for a lower frequency of "family history of OA" in dropouts due to missing data (38\% vs 49\%). One could debate whether this difference is clinical relevant. An association between development of KOA and family history of OA has been described; ${ }^{33}$ however, others could not confirm this. ${ }^{34}$ Overall, we estimated the possibility of selection bias as minimal. As a solution for the missing data, multiple imputation was considered. However, as described in a article by Von Hippel et al, ${ }^{35}$ multiple imputation is only the solution when the independent variables are missing at random. When the outcome is missing and the independent variables are complete, as in our study, the incomplete cases contribute no information to the outcome estimate and would only add noise to these estimates. ${ }^{35}$ Second, a possible disadvantage of using an randomized controlled trial for prediction modeling is that there are set points for follow-up measurements, in the present study after 2.5 and 6.5 years. In this way, no difference can be made between participants who develop knee pain after a short or after a longer follow-up time. Ultimately, there might have been more use from a model that took "time to development of FKP/KOA" as the outcome of interest, but these data were not available. Further, the overlap between the prediction of both outcomes is large, since age $>50$ years and FKP are part of the definition of the combined ACR criteria. ${ }^{17}$ To make prediction applicable to a GP's daily practice, we presented the symptom-only definition in the main text and the official definition for symptomatic KOA in the Appendix. By analyzing data on subject level, with the aim to predict for a person and not for a knee, details on knee level are lost. As a consequence, it might be possible that presented symptoms were in the other knee than the outcome. Significant associations might be noncausal. Since proving causality is not the aim in prediction analysis, ${ }^{36}$ this seems not to affect results.

\section{Implications for Research and/or Practice}

Although incidence rates were relatively high after 6.5 years, the majority of women were free of FKP and symptomatic KOA. It might be worthwhile to obtain other variables that can discriminate in high-risk subjects. We did not include, for instance known risk factors for incident knee OA like malalignment, meniscal damage or effusion synovitis. ${ }^{37-39}$ Those measures are obtained by radiologic assessments and not directly applicable in a GP's office. Surprisingly, no literature is available about the diagnostic accuracy of the clinical diagnosis of malalignment by a GP or the validity of knee specific questions for the diagnosis of synovitis. Diagnostic accuracy of the McMurray or Apley test for meniscal damage is small and not advised in general practice. ${ }^{40}$ It seems necessary to improve the clinical diagnosis of the above risk factors in general practice or search for others.

This study showed that in middle-aged overweight women in general practice, the use of easily obtainable risk factors contributed moderately to prediction of FKP and symptomatic KOA. Since the discriminative ability of the prediction models was moderate, the prediction of FKP and symptomatic KOA seems not yet clinically applicable.

The authors thank all the participants and staff of the Prevention of Knee Osteoarthritis in Overweight Females study. The authors also thank Diana van Emmerik, BSc for her efforts in the data collection and Yasmaine Karel, MSc and Arianne Verhagen, PhD for their help with the statistical analysis.

To see this article online, please go to: http://jabfm.org/content/ 32/4/575.full.

\section{References}

1. Peat G, McCarney R, Croft P. Knee pain and osteoarthritis in older adults: A review of community burden and current use of primary health care. Ann Rheum Dis 2001;60:91-7. 
2. McAlindon TE, Cooper C, Kirwan JR, et al. Knee pain and disability in the community. Br J Rheumatol 1992;31:189-92.

3. Bedson J, Croft PR. The discordance between clinical and radiographic knee osteoarthritis: A systematic search and summary of the literature. BMC Musculoskelet Disord 2008;9:116.

4. Hunter DJ, Schofield D, Callander E. The individual and socioeconomic impact of osteoarthritis 2014; 10:437-41.

5. Blagojevic M, Jinks C, Jeffery A, et al. Risk factors for onset of osteoarthritis of the knee in older adults: A systematic review and meta-analysis. Osteoarthritis Cartilage 2010;18:24-33.

6. Sheeran P, Harris PR, Epton T. Does heightening risk appraisals change people's intentions and behavior? A meta-analysis of experimental studies. Psychol Bull 2014;140:511-43.

7. Marteau TM, French DP, Griffin SJ, et al. Effects of communicating DNA-based disease risk estimates on risk-reducing behaviours. Cochrane Database Syst Rev 2010;(10):CD007275.

8. Smerecnik C, Grispen JE, Quaak M. Effectiveness of testing for genetic susceptibility to smoking-related diseases on smoking cessation outcomes: A systematic review and meta-analysis. Tob Control 2012;21: 347-54.

9. Kerkhof HJ, Bierma-Zeinstra SM, Arden NK, et al. Prediction model for knee osteoarthritis incidence, including clinical, genetic and biochemical risk factors. Ann Rheum Dis 2014;73:2116-21.

10. Zhang W, McWilliams DF, Ingham SL, et al. Nottingham knee osteoarthritis risk prediction models. Ann Rheum Dis 2011;70:1599-604.

11. Sharma L, Hochberg M, Nevitt M, et al. Knee tissue lesions and prediction of incident knee osteoarthritis over 7 years in a cohort of persons at higher risk. Osteoarthritis Cartilage 2017;25:1068-75.

12. Kellgren JH, Lawrence JS. Radiological assessment of osteo-arthrosis. Ann Rheum Dis 1957;16:494502.

13. Runhaar J, van Middelkoop M, Reijman M, et al. Prevention of knee osteoarthritis in overweight females: The first preventive randomized controlled trial in osteoarthritis. Am J Med 2015;128:88895.e4.

14. Silverwood V, Blagojevic-Bucknall M, Jinks C, et al. Current evidence on risk factors for knee osteoarthritis in older adults: A systematic review and metaanalysis. Osteoarthritis Cartilage 2015;23:507-15.

15. Symmons DP. Knee pain in older adults: The latest musculoskeletal "epidemic". Ann Rheum Dis 2001; 60:89-90.

16. Hadler NM. Knee pain is the malady-Not osteoarthritis. Ann Intern Med 1992;116:598-9.

17. Altman R, Asch E, Bloch D, et al. Diagnostic and Therapeutic Criteria Committee of the American Rheumatism Association. Development of criteria for the classification and reporting of osteoarthritis. Classification of osteoarthritis of the knee. Arthritis Rheum 1986;29:1039-49.

18. Buckland-Wright JC, Wolfe F, Ward RJ, et al. Substantial superiority of semiflexed (MTP) views in knee osteoarthritis: A comparative radiographic study, without fluoroscopy, of standing extended, semiflexed (MTP), and schuss views. J Rheumatol 1999;26:2664-74.

19. McWilliams DF, Leeb BF, Muthuri SG, et al. Occupational risk factors for osteoarthritis of the knee: A meta-analysis. Osteoarthritis Cartilage 2011;19: 829-39.

20. Jinks C, Jordan KP, Blagojevic M, et al. Predictors of onset and progression of knee pain in adults living in the community. A prospective study. Rheumatology (Oxford) 2008;47:368-74.

21. Schiphof D, van Middelkoop M, de Klerk BM, et al. Crepitus is a first indication of patellofemoral osteoarthritis (and not of tibiofemoral osteoarthritis). Osteoarthritis Cartilage 2014;22:631-8.

22. Peat G, Thomas E, Duncan R, et al. Clinical classification criteria for knee osteoarthritis: performance in the general population and primary care. Ann Rheum Dis 2006;65:1363-7.

23. Roos EM, Roos HP, Lohmander LS, Ekdahl C, Beynnon BD. Knee Injury and Osteoarthritis Outcome Score (KOOS)—Development of a self-administered outcome measure. J Orthop Sports Phys Ther 1998;28:88-96.

24. Ikram MA, Brusselle GGO, Murad SD, et al. The Rotterdam Study: 2018 update on objectives, design and main results. Eur J Epidemiol 2017;32:807-50.

25. Wendel-Vos GC, Schuit AJ, Saris WH, Kromhout D. Reproducibility and relative validity of the short questionnaire to assess health-enhancing physical activity. J Clin Epidemiol 2003;56:1163-9.

26. Hensor EM, Dube B, Kingsbury SR, et al. Data from the Osteoarthritis Initiative. Toward a clinical definition of early osteoarthritis: Onset of patient-reported knee pain begins on stairs. Data from the Osteoarthritis Initiative. Arthritis Care Res (Hoboken) $2015 ; 67: 40-7$.

27. Cooper C, McAlindon T, Coggon D, et al. Occupational activity and osteoarthritis of the knee. Ann Rheum Dis 1994;53:90-3.

28. O'Reilly SC, Muir KR, Doherty M. Occupation and knee pain: A community study. Osteoarthritis Cartilage 2000;8:78-81.

29. Luyten FP, Bierma-Zeinstra S, Dell'Accio F, et al. Toward classification criteria for early osteoarthritis of the knee. Semin Arthritis Rheum 2018;47: 457-63.

30. Felson DT, Zhang Y, Hannan MT, et al. The incidence and natural history of knee osteoarthritis in the elderly. The Framingham Osteoarthritis Study. Arthritis Rheum 1995;38:1500-5. 
31. Leyland KM, Hart DJ, Javaid MK, et al. The natural history of radiographic knee osteoarthritis: A fourteen-year population-based cohort study. Arthritis Rheum 2012;64:2243-51.

32. Cooper C, Snow S, McAlindon TE, et al. Risk factors for the incidence and progression of radiographic knee osteoarthritis. Arthritis Rheum 2000;43:995-1000.

33. Huétink K, Stoel BC, Watt I, et al. Identification of factors associated with the development of knee osteoarthritis in a young to middle-aged cohort of patients with knee complaints. Clin Rheumatol 2015;34:1769-79.

34. Riyazi N, Meulenbelt I, Kroon HM, et al. Evidence for familial aggregation of hand, hip, and spine but not knee osteoarthritis in siblings with multiple joint involvement: the GARP study. Ann Rheum Dis 2005;64:438-43.

35. Von Hippel PT. Regression with missing ys: An improved strategy for analyzing multiply imputed data. Sociol Methodol 2007;37:83-117.
36. Shmueli G. To explain or to predict? Stat Sci 2010; 25:289-310.

37. Atukorala I, Kwoh CK, Guermazi A, et al. Synovitis in knee osteoarthritis: A precursor of disease? Ann Rheum Dis 2016;75:390-5.

38. Felson DT, Niu J, Gross KD, et al. Valgus malalignment is a risk factor for lateral knee osteoarthritis incidence and progression: Findings from the Multicenter Osteoarthritis Study and the Osteoarthritis Initiative. Arthritis Rheum 2013;65:355-62.

39. Englund M, Roemer FW, Hayashi D, Crema MD, Guermazi A. Meniscus pathology, osteoarthritis and the treatment controversy. Nat Rev Rheumatol 2012;8:412-9.

40. Karachalios T, Hantes M, Zibis AH, et al. Diagnostic accuracy of a new clinical test (the Thessaly test) for early detection of meniscal tears. J Bone Joint Surg Am 2005;87:955-62. 
Appendix Table 1. Multivariable Models in Prediction of Incident Symptomatic Knee Osteoarthritis (OA) and Internal Validation (Calibration and Discrimination) of the Risk Prediction Models

\begin{tabular}{|c|c|c|c|c|}
\hline \multirow[b]{2}{*}{ Selected Predictors } & \multicolumn{2}{|c|}{ Basic Model } & \multicolumn{2}{|c|}{ Backward Model } \\
\hline & OR $(95 \% \mathrm{CI})$ & $P$ Value & OR $(95 \% \mathrm{CI})$ & $P$ Value \\
\hline Age (years) & $1.07(0.98-1.17)$ & .15 & $1.14(1.02-1.28)$ & .02 \\
\hline BMI $\left(\mathrm{kg} / \mathrm{m}^{2}\right)$ & $1.12(1.04-1.20)$ & $<.01$ & $1.16(1.06-1.26)$ & .001 \\
\hline Mild knee symptoms* & & & $1.73(0.85-3.49)$ & .13 \\
\hline Knee problems while climbing stairs ${ }^{\dagger}$ & & & $2.08(1.03-4.23)$ & .04 \\
\hline Morning stiffness ${ }^{\ddagger}$ & & & $2.46(0.97-6.25)$ & .06 \\
\hline Postmenopausal status & & & $0.54(0.26-1.12)$ & .10 \\
\hline Physically demanding work ${ }^{\S}$ & & & $2.38(0.83-6.83)$ & .11 \\
\hline AUC of the model & $0.63(0.54-0.71)$ & & $0.72(0.64-0.80)$ & \\
\hline Calibration: Hosmer-Lemeshow $P$ value & .96 & & .20 & \\
\hline Variance explained (Nagelkerke), \% & 6.5 & & 21.9 & \\
\hline
\end{tabular}

AUC, area under the curve of the receiver operating characteristic; BMI, body mass index; CI, confidential interval; OR, odds ratio. Bold indicates $P<.05$.

*Pain in or around the knee within the last 12 months.

${ }^{\dagger}$ Defined with the Knee Injury and Osteoarthritis Outcome Score (KOOS) on physical functioning.

${ }^{\ddagger}$ Defined with the KOOS on stiffness.

${ }^{\S}$ Defined as doing intense work (regularly lifting heavy objects at work).

Appendix Table 2. External validation for the prediction of incident symptomatic knee Osteoarthritis (OA) in Rotterdam Study-III-1

\begin{tabular}{|c|c|c|}
\hline \multirow[b]{2}{*}{ Selected Predictors } & \multicolumn{2}{|c|}{ Study Population $(\mathrm{n}=264)$} \\
\hline & OR $(95 \% \mathrm{CI})$ & $P$ Value \\
\hline Age (years) & $1.13(0.93-1.37)$ & .22 \\
\hline $\mathrm{BMI}\left(\mathrm{kg} / \mathrm{m}^{2}\right)$ & $1.09(0.97-1.22)$ & .15 \\
\hline Mild knee symptoms* & $1.53(0.49-5.08)$ & .44 \\
\hline Knee problems while climbing stairs ${ }^{\dagger}$ & $4.47(1.31-15.23)$ & .02 \\
\hline Morning stiffness ${ }^{\ddagger}$ & $2.14(0.68-6.73)$ & .19 \\
\hline Postmenopausal status & $1.33(0.22-7.98)$ & .76 \\
\hline Physically demanding work ${ }^{\S}$ & $1.50(0.50-4.53)$ & .48 \\
\hline AUC of the model & $0.81(0.71-0.90)$ & \\
\hline Calibration: Hosmer-Lemeshow $P$ value & .17 & \\
\hline Variance explained (Nagelkerke), \% & 21.9 & \\
\hline
\end{tabular}

AUC, area under the curve of the receiver operating characteristic; BMI, body mass index; CI, confidential interval; OR, odds ratio. Bold indicates $P<.05$.

*Pain in or around the knee within the last 12 months.

${ }^{\dagger}$ Defined with the Knee Injury and Osteoarthritis Outcome Score (KOOS) on physical functioning.

${ }^{\ddagger}$ Defined with the KOOS on stiffness.

§Defined as doing intense work (regularly lifting heavy objects at work). 